\title{
EL SOFTWARE LIBRE EN LAS EMPRESAS DE MONTERÍA
}

\author{
Juan Vergara-Villadiego ${ }^{1}$, Francisco Torres-Hoyos ${ }^{2}$
}

${ }^{1}$ Docente investigador del programa de Ingeniería de Sistemas, Universidad Cooperativa de Colombia

Correo electrónico: juan.vergara@campusucc.edu.co

${ }^{2}$ Docente investigador del departamento de Física, Universidad de Córdoba y Universidad Cooperativa de Colombia

Montería, Colombia

Recibido: 12 de junio del 2014. Aprobado: 12 de octubre del 2014.

Cómo citar este artículo: J. Vergara-Villadiego y F. Torres-Hoyos, "El software libre en las empresas de Montería". Ingeniería Solidaria, vol. 10, n. 17, pp. 105-110, en.-dic., 2014. doi: http://dx.doi.org/10.16925/in.v9i17.810

Resumen. Según el registro de la Cámara de Comercio, de las 170 empresas registradas en Montería, el 98,2\% corresponden a micros, pequeñas y medianas empresas (Mipymes). Los autores seleccionaron una muestra representativa de 50 empresas que corresponden al 30\% de las empresas legalmente registradas en Montería. Los casos estudiados se caracterizan por ser Mipymes dedicadas a la venta de servicios de sistemas informáticos y aplicaciones de software. Para el estudio se diseñaron 2 encuestas de tipo evaluativo. Los resultados señalan que un 95,6\% de las Mipymes usan software con licencia propietaria, sin contar con el debido licenciamiento; el 90,4\% no utiliza software libre y, además, el 75\% confunde software libre con gratis. Los autores explican cómo realizaron un diagnóstico del uso y conocimiento del software libre en las Mipymes de Montería.

Palabras clave: software libre, software propietario, licencia, piratería, aplicaciones de software, derecho de autor.

\section{Free Software at Companies in Montería}

Abstract. According to the Chamber of Commerce registry, $98.2 \%$ of the 170 companies registered in Montería are micro, small and mediumsized enterprises (MSMEs). The authors selected a representative sample of 50 companies that account for $30 \%$ of those legally registered in Montería. These cases are MSMEs that sell informatics systems and software applications. 2 evaluative type surveys were designed for the study. The results show that $95.6 \%$ of the MSMEs use proprietary software without actually having the proper licenses; $90.4 \%$ do not use free software and $75 \%$ confuse free software with software that is free of charge. The authors explain how they examined the use and knowledge of free software at the MSMEs of Montería.

Keywords: free software, proprietary software, license, piracy, software applications, copyright.

\section{O SOFTWARE LIVRE NAS EMPRESAS DE MONTERÍA}

Resumo. Segundo o registro da Câmara do Comércio, das 170 empresas registradas em Montería (Colômbia), 98,2\% correspondem a micros, pequenas e médias empresas (PME). Os autores selecionaram uma amostra representativa de 50 empresas que correspondem a $30 \%$ das empresas legalmente registradas em Montería. Os casos estudados se caracterizam por serem PME dedicadas à venda de serviços de sistemas informáticos e aplicações de software. Para o estudo, foram desenhadas duas enquetes de tipo avaliativo. Os resultados indicam que $95,6 \%$ das PME usam software com licença proprietária, sem contar com o devido licenciamento; $90,4 \%$ não utilizam software livre e, além disso, $75 \%$ confundem software livre com gratuito. Os autores explicam como realizaram um diagnóstico do uso e conhecimento do software livre nas PME de Montería.

Palavras-chave: software livre, software proprietário, licença, pirataria, aplicações de software, direitos autorais. 


\section{Introducción 1}

El software libre es un movimiento exitoso debido a su alto impacto en todos los sectores de la sociedad, tal como se evidencia en proyectos de Android, Linux, Libre Office, Apache, Gráficos de Red Portátiles png, entre otros. Además, es un modelo innovador que fomenta el espíritu de colaboración y la libertad. En este trabajo, se pretende crear un marco de referencia que ayude a identificar las aplicaciones de software utilizadas, su estado de licenciamiento y el conocimiento acerca del software libre.

Las Mipymes de la ciudad de Montería son empresas que, a nivel organizativo y tecnológico, no cuentan con planes claros para la adaptación a las Tecnologías de la Información y Comunicaciones (TIC), que les permitan crecer adecuadamente.

Se seleccionaron para este estudio 50 Mipymes mediante el método de muestreo aleatorio estratificado con fijación proporcional. A los casos seleccionados se les aplicaron dos instrumentos diseñados para la recolección de información. El primero de ellos se implementó con el propósito de tener un inventario tecnológico, tanto a nivel de hardware como de software, de las empresas, así como para tener información del estado del licenciamiento de las mismas. El segundo instrumento fue diseñado para obtener información acerca del conocimiento de software libre, sus ventajas y grado de utilización; asimismo, se evaluó el conocimiento de las leyes de derechos de autor y las posibles sanciones en las que se puede incurrir cuando se utiliza cualquier aplicación informática sin su debido licenciamiento.

El artículo está organizado de la siguiente manera: en la sección 2 , se presentan los materiales y métodos; los resultados y discusión, en la sección 3 y las conclusiones, en la sección 4.

\section{Materiales y métodos}

En los numerales 2.1 y 2.2 se describen de forma concisa los instrumentos de evaluación y procesamiento de la información recolectada.

1 Este artículo es producto de la investigación "El software libre: una alternativa para reducir el uso de software no licenciado en las Mipymes de Montería", aprobado por el Comité Nacional de Investigación CONADI con vigencia del 2014.

\subsection{Diseño de instrumentos de evaluación}

Se elaboraron y aplicaron dos instrumentos consultivos para conocer el tipo de aplicaciones de software utilizadas por las Mipymes de Montería, su conocimiento acerca de software libre, licenciamiento y derechos de autor.

El primer instrumento contiene 10 preguntas que nos permiten recoger información sobre el estado actual, a nivel de aplicaciones, de los software utilizados teniendo en cuenta su clasificación (véase figura 1), es decir, divididos entre sistemas operativos (servidor y escritorio), paquetes ofimáticos, navegadores, utilidades y aplicaciones dirigidas a la gestión de la empresa como paquetes contables y sistema de información de toma de decisiones (anexo 1). En el segundo instrumento, se tienen también 10 preguntas que indagan sobre el conocimiento del software libre, las ventajas que ofrece a nivel organizacional y los posibles impedimentos para una implantación (anexo 2).

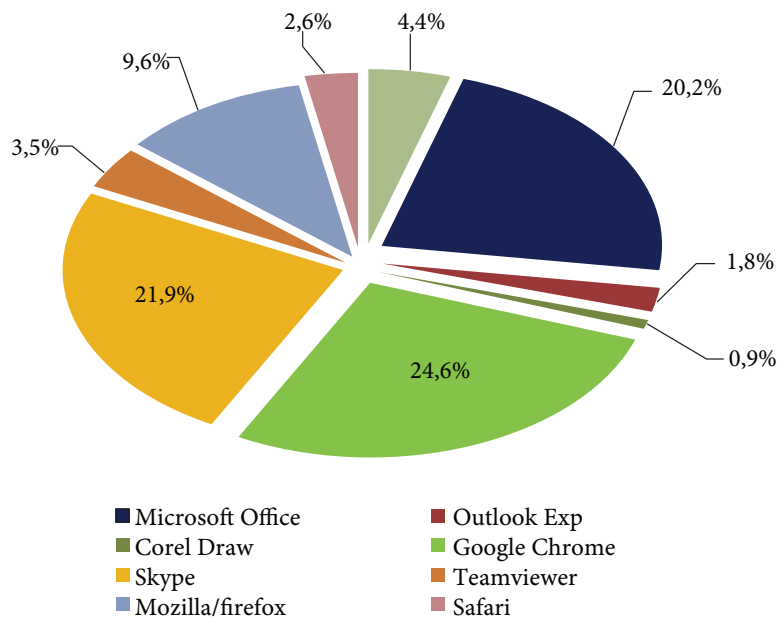

Figura 1. Índices de aplicaciones de escritorio utilizadas Fuente: elaboración propia

\subsection{Procesamiento de la información}

El análisis estadístico de la información recolectada por medio de los instrumentos aplicados se realizó con el Programa para el Análisis Estadístico de Datos Muestreados (PSPP, por sus siglas en inglés), el cual permite llevar a cabo procedimientos estadísticos rápidos en modos terminal e interfaz gráfica; soporta más de mil millones de variables; es compatible con proyectos como Gnumeric y OpenOffice, y es multiplataforma, lo que permite instalarlo en múltiples sistemas operativos [1]. Por estas razones, lo consideramos pertinente para este trabajo. 


\subsubsection{Selección de la muestra}

Teniendo en cuenta la distribución estadística que presentan las 170 Mipymes de ventas de servicio, se utilizó el método de muestreo aleatorio estratificado con afijación proporcional. El método, expresado en la ecuación 1, sirvió para determinar el tamaño de la muestra representativa con una confiabilidad del 90\%, esto es:

$$
n_{i}=\frac{\sum W_{h} P_{h} Q_{h}}{\frac{E^{2}}{Z^{2}}} ; n=\frac{n_{i}}{1+\frac{n_{i}}{N}}
$$

Donde

$W_{h}=$ Proporción de empresas del sector seleccionado

$P_{h}=0,5$ (probabilidad de que cumpla la condición de estudio (utilización de software libre)

$Q_{h}=0,5$ (probabilidad de que no utilice software libre)

$E=0,098$ (error de muestreo)

$Z=1,65$ (percentil de confiabilidad de la muestra de estudio)

$N=$ Número total de empresas seleccionadas

$n_{\mathrm{i}}=$ Muestra para población infinita

$n=$ Muestra ajustada a la población seleccionada

\section{Resultados y análisis}

\subsection{Tamaño muestral}

Teniendo en cuenta los parámetros establecidos para el muestreo y al aplicar la ecuación 1, se calculó el valor de la muestra representativa de la población en estudio, la cual fue de 50 .

\subsection{Aplicaciones de escritorio}

Se aplicó el instrumento de medición a las Mipymes (anexo 1) y los resultados obtenidos para las aplicaciones de escritorio se presentan en la figura 1 , donde se ve que los paquetes ofimáticos representan un $20,2 \%$; las aplicaciones de mensajería 21,9\%; los clientes de correo electrónico un 1.8\%; las utilidades 3,5\%; los navegadores un $41,2 \%$ y los software para diseño asistido un $0,9 \%$. De las aplicaciones utilizadas, solo Mozilla/ Firefox, con un 9,6\%, representa el uso de software libre, lo cual es un porcentaje bajo con relación al uso del producto en otros países [2].

Con respecto a Microsoft Office, el 95,6\% de las Mipymes, no cuenta con el debido licenciamiento, de manera que cumplen con este solo el 4,4\%. En este sen- tido, no se evidencia ningún paquete ofimático de licencia libre, tal como se muestra en la figura 2.

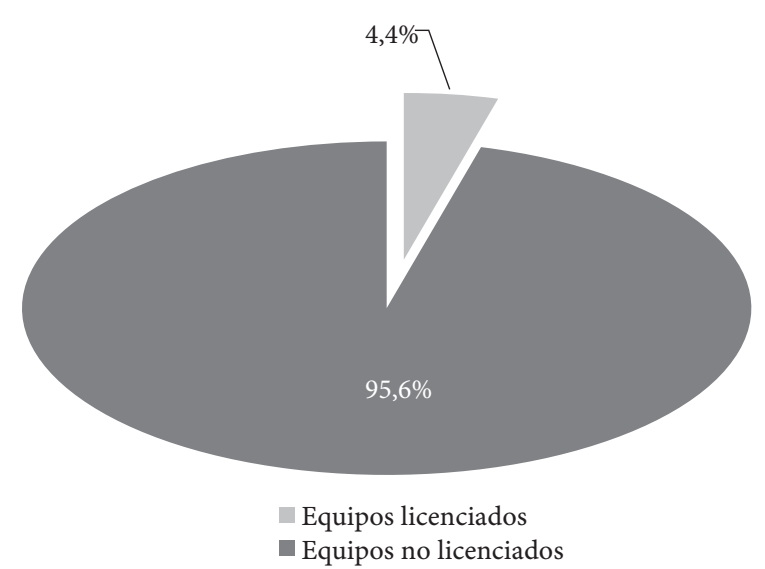

Figura 2. Índices de licenciamiento Microsoft Office Fuente: elaboración propia

Es claro, entonces, que el grado de ilegalidad en el cual están incurriendo las empresas antes mencionadas es bastante significativo, lo que puede generar sanciones por parte de las entidades encargadas de ejercer controles de protección a la propiedad intelectual [3].

En cuanto a los sistemas operativos utilizados en computadoras de escritorio y portátiles (anexo 1), se obtuvo como resultado que el $72 \%$ corresponde a Windows 7, el 25\% a Windows XP y un 3\% a Mac OS, como se muestra en la figura 3.

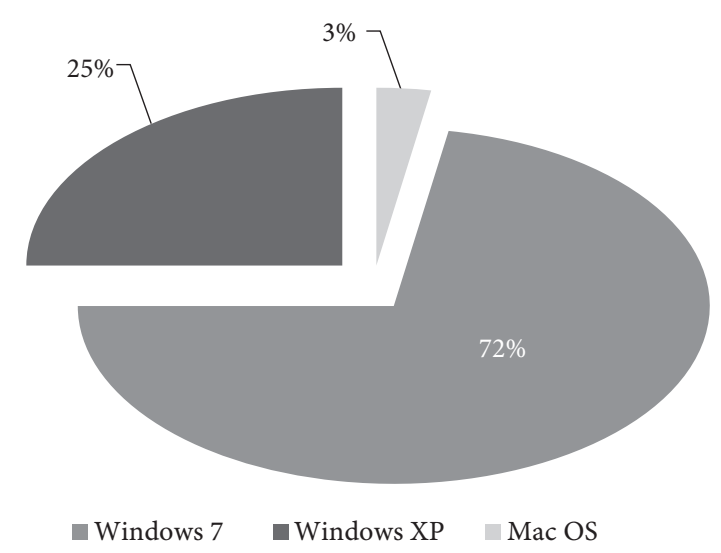

Figura 3. Índices de uso Sistemas Operativos Microsoft Fuente: elaboración propia

Como se puede observar en la figura 3, el mayor porcentaje de los sistemas operativos usados por las Mipymes son de Microsoft y, basado en ello, se quería ver su estado de licenciamiento, cuyos resultados se presentan en la figura 4. 


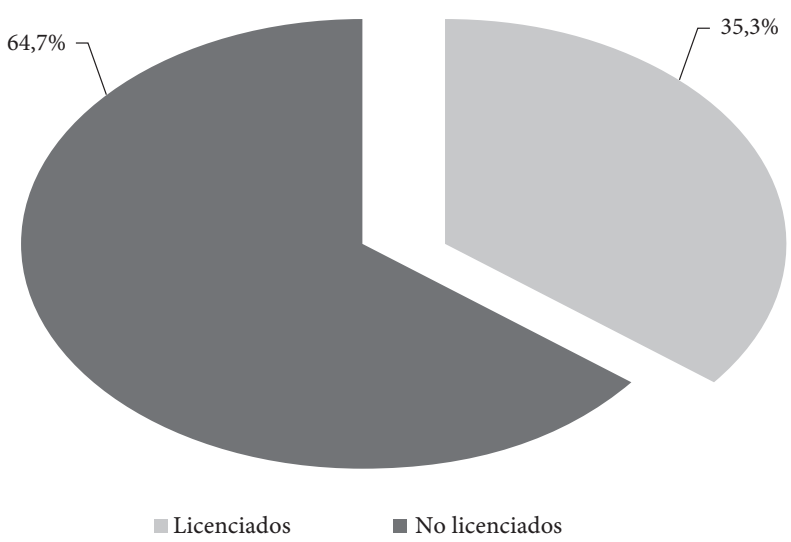

Figura 4. Índices de licenciamento de Sistemas Operativos (Microsoft)

Fuente: elaboración propia

La figura 4 muestra que un $64,7 \%$ de los sistemas operativos utilizados no cuentan con licencia y solamente un $35,3 \%$ la posee. De los no licenciados, el $47,9 \%$ corresponde a Windows 7 y $16,8 \%$, a Windows XP; mientras que los licenciados, el $24.4 \%$ corresponden a Windows 7 ; el $8,4 \%$, a XP, y el 2,5\% a Mac OS. Como se puede apreciar en esta estadística, no existe un sistema operativo de licencia libre que sea utilizado por las Mipymes.

\subsection{Licenciamiento de software}

En este sentido se hizo una pregunta consultiva (anexo 1 ), cuyos resultados son expresados en la figura 5.

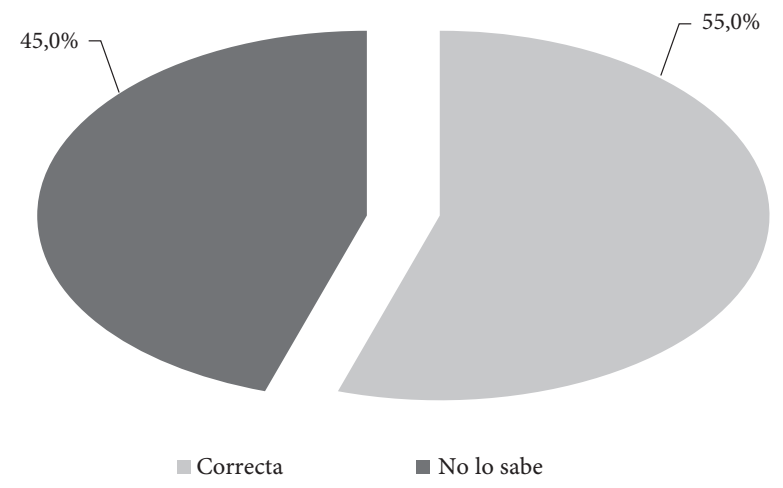

Figura 5. Conocimiento de licencias de software Fuente: elaboración propia

Como se puede ver en la figura 5, un 55\% de los propietarios de las Mipymes conocen sobre el licenciamiento de software, mientras que el $45 \%$ afirma desconocerlo en gran parte. Lo anterior nos indica que casi la mitad de los propietarios de las Mipymes adolecen del conocimiento explícito del licenciamiento de software, lo que puede conducir a conductas punibles. Es por ello que se necesita de una cualificación seria y responsable por parte de las entidades gubernamentales, bien sea del orden municipal, departamental o nacional, sobre el licenciamiento de software en Montería. Lo anterior, aparte de conducir al conocimiento en pleno de las leyes que rigen a la propiedad intelectual -de acuerdo con lo establecido en el artículo 61 de la Constitución Política de Colombia [4]- también deberá ser un gran incentivo a la cultura de la legalidad.

\subsection{Software libre}

Se encuestó, con base en el anexo 2, qué porcentaje de conocimiento tenían los empresarios de Montería sobre software libre y específicamente sobre el concepto del mencionado, obteniéndose los siguientes resultados (figura 6).

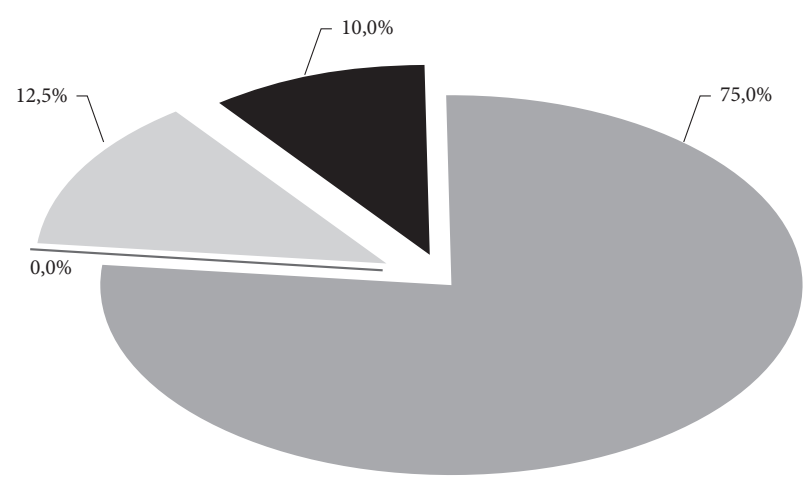

$$
\begin{aligned}
& \text { - Sí, es gratis } \\
& \text { - Sí, es un software con restricciones } \\
& \text {-Sí, es un software sin restricciones } \\
& \text { - No lo se }
\end{aligned}
$$

Figura 6. Conocimiento del término de software libre Fuente: elaboración propia

En la figura 6 se puede ver que el 75\% de los propietarios aseguran conocer el término software libre, asociándolo con gratuidad. Un $12,5 \%$ afirma que conoce el término y lo distinguen como un software sin restricciones; el 10\% manifiesta no conocerlo y nadie lo identifica como un software con restricciones. Lo anterior conduce a afirmar que el término software libre es asumido simplemente como algo gratuito o, en su defecto, con software que se puede utilizar sin pagar. Probablemente, el origen de tal confusión radica en la ambigüedad al traducir al español el término inglés "free", que significa libre y también gratis, pero, en realidad, a lo que se hace alusión con el término software 
libre, tal como fue concebido por Richard Stallman en su definición Free Software Foundation, "Free software definition" [5], hace referencia a las libertades que puede ejercer quien lo recibe, las cuales son:

1. Libertad para ejecutar el programa en cualquier sitio, con cualquier propósito y para siempre;

2. Libertad para estudiarlo y adaptarlo a nuestras necesidades. Esto exige el acceso al código fuente;

3. Libertad de redistribución, de modo que se nos permita colaborar con vecinos y amigos, $y$

4. Libertad para mejorar el programa y publicar sus mejoras. Esto también exige el código fuente [6].

Ahora bien, cuando se consultó sobre los motivos por los cuales las Mipymes no utilizan software libre (anexo 2), obtuvimos los resultados que se presentan en la figura 7.

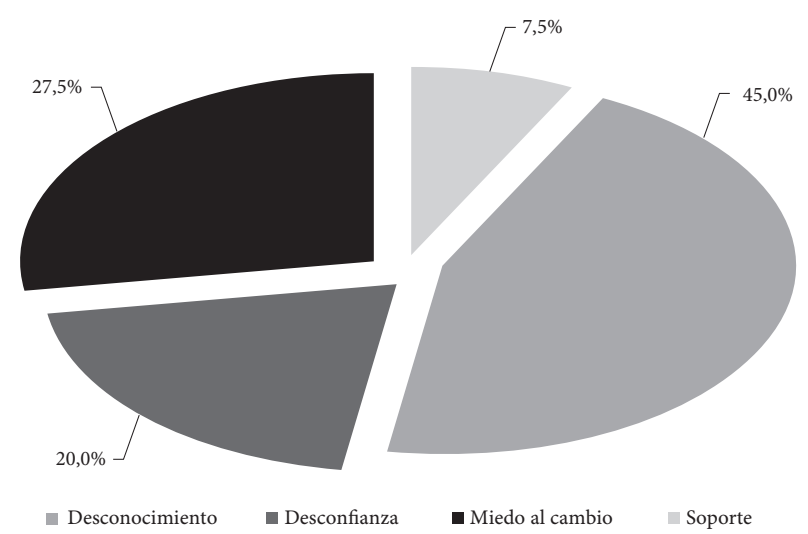

Figura 7. Motivos de la no adopción del software libre Fuente: elaboración propia

La figura anterior muestra que un $45 \%$ de los propietarios demuestra falta de conocimiento para implantar software libre; un 27,5\%, miedo al cambio; un $20 \%$, desconfianza, y un $7,5 \%$, falta de soporte.

Al observar los distintos motivos reflejados en la estadística inmediatamente anterior, podemos afirmar que existe la necesidad de realizar actividades de capacitación y divulgación sobre software libre con el fin de mostrar a las Mipymes que existen alternativas para disminuir el alto índice de piratería, sin incurrir en gastos por concepto de licenciamiento.

\section{Conclusiones}

De acuerdo con los resultados mostrados en la figura 2, existe un alto índice de software propietario no licenciado en las Mipymes de Montería, ya que un $95,6 \%$ de los paquetes ofimáticos no poseen licencia. Igual ocurre con los sistemas operativos con un porcentaje del 64,7\%, como se aprecia en la figura 4. Estos índices se encuentran muy elevados si se comparan con los resultados publicados por la Alianza del Software de Negocios (BSA, por su sigla en inglés) para el resto de Latinoamérica [7]. Existen, sin embargo, particularidades que pueden explicar lo anterior. El fenómeno de la piratería, por ejemplo, tiene un gran arraigo social, económico y legal: sus posibles causas pueden ser desde la escasa sensibilidad de los empresarios, la protección ineficaz de la propiedad intelectual, hasta el precio elevado de las licencias de software.

Basados en la figura 5, podemos decir que algo más de la mitad de los propietarios de las Mipymes conocen acerca del licenciamiento de software (exactamente el 55\%), pero el resto, es decir, el $45 \%$, manifiestan no conocerlo en detalle. Esto último puede ser un pretexto para incurrir en la ilegalidad, por tanto creemos conveniente y necesario realizar una capacitación masiva, de carácter obligatorio, a los propietarios de empresas en Montería, cuyo objeto sea el licenciamiento de software, para con ello dejar claro cuáles son los delitos en que se incurre y, por ende, cuáles serían sus sanciones [8].

Es evidente en la figura 6 la confusión entre software libre y gratis, ya que el $75 \%$ de los empresarios encuestados así lo puso de manifiesto, cuando en realidad a lo que se hace alusión es a la libertad para ejecutar, estudiarlo, redistribuirlo y mejorar el programa.

A pesar de que los propietarios de las Mipymes en un $45 \%$ aduce falta de conocimiento para implantar software libre, un $27,5 \%$ miedo al cambio, un $20 \%$ desconfianza y un $7,5 \%$ por falta de soporte, el software libre se empieza a ver como una herramienta que podría tender puentes sobre las brechas de acceso a las TIC, además conlleva obtener ventajas tales como: independencia tecnológica, reducción de costos, apropiación de conocimiento, transparencia y seguridad [9].

El desconocimiento de utilizar una aplicación licenciada sin autorización para ello, por parte de los propietarios de las Mipymes, ocasiona violar las leyes de propiedad intelectual y expone a riegos la información de la empresa, entre otros. Es aquí donde el software libre juega un papel fundamental y se convierte en una alternativa para optar por la Mipyme, para reemplazar las aplicaciones propietarias no licenciadas por aplicaciones equivalentes con licencia libre. 
Por último, se hace necesario un trabajo interdisciplinario por parte de las entidades de control, empresas, universidades, Alcaldía y Gobernación de Córdoba para crear oficinas o consultorios de asesoría tecnológica, que ayuden a tomar conciencia a las Mipymes, de la importancia que tienen las TIC en la competitividad de las empresas, generando para ello programas de educación tecnológica con acompañamiento constante y profesional, que conlleve la elaboración de un plan estratégico de sistemas a partir de un diagnóstico inicial [10].

Otro aspecto importante es que, en Colombia, no existen políticas que promocionen o impulsen el uso de software libre en ningún ámbito, tampoco campañas educativas acerca de licenciamiento de software y derechos de autor, en comparación con otros países como Venezuela, España, Ecuador o Cuba, que han emitido leyes donde acogen el software libre para su utilización y desarrollo en entidades públicas, instituciones educativas y universidades estatales [11].

\section{Agradecimientos}

Los autores agradecen a la Universidad Cooperativa de Colombia el apoyo económico para el desarrollo de este trabajo.

\section{Referencias}

[1] GNU Operating System. GNU PSPP. (2013) [En línea]. Disponible en: http://www.gnu.org/software/ pspp/, último acceso: sept. 10, 2014.

[2] S. Luján Mora y J. Aragonés Ferrero, “6 estadísticas de uso de navegadores”, Nuevos estándares en el desarrollo de sitios web: HTML5 y CSS3, 2012 [En línea]. Disponible en: http://desarrolloweb.dlsi.ua.es/cursos/2012/ nuevos-estandares-desarrollo-sitios-web/estadisticasuso-navegadores, último acceso: agosto 12, 2014].

[3] J. A. Pabón Cadavid, Guía de derecho de autor para bibliotecas. Bogotá: CTP, 2010.

[4] República de Colombia, Constitución Política de Colombia. Bogotá: Ed. Leyer, 2013, p. 10

[5] R. M. Stallman, Software libre para una sociedad libre (Introducción de Lawrence Lessig). Madrid: GNU Press, 2002, p. 45.

[6] J. M. González Barahona, J. Seoane Pascual y G. Robles, Software Libre. Cataluña: UOC, 2012.

[7] Business Software Alliance, Ninth Annual BSA Global Software 2011 Piracy Study. [En línea]. Disponible en: http://globalstudy.bsa.org/2011/, último acceso: septiembre 10, 2014

[8] Congreso de Colombia (2006, Junio 22). Ley 1032 de 2006, por la cual se modifican los artículos 257, 271, 272 y 306, art. 271. [En línea]. Disponible en: http://www. camlibro.com.co/pdf/ley_1032_2006.pdf

[9] L. Zúñiga, El software libre y sus perspectivas para el desarrollo en América Latina y el Caribe. Bellanet International Secretariat, 2004, p. 14. [En línea]. Disponible en: http://www.sulabatsu.com/ voces/Documentos/revisionbiblio.pdf, último acceso: septiembre 1, 2014.

[10] CCIT y Fedesarrollo, El papel de las tic en el desarrollo de la pequeña empresa: reflexiones de política a la luz del caso colombiano. Bogotá: CCIT, 2013. [En línea]. Disponible en: http://www.fedesarrollo.org.co/wp-content/uploads/2013/12/TIC_diciembre_2013.pdf

[11] A. Abella, J. Sánchez y M. A. Segovia, Libro blanco del software libre en España. Segovia: Ed. A. Abella, J. Sánchez y M. A, 2004. 